\title{
Construal level as a mediator of stress-induced bias in financial decision making
}

\author{
Sekoul Krastev; Dan Pilat; Matthew Martin; Marielle C. Montenegro; David Brooke Struck ${ }^{\mathrm{a}}$ \\ The Decision Lab
}

(Dated: January 16, 2020)

\begin{abstract}
Stress is extremely prevalent yet poorly understood in the context of decision making. While we know that high levels of stress can impact the distribution of cognitive resources, it is unclear how this is manifested in a complex decision-making context and what, if any, strategies can be leveraged to optimize stressed decisions. Financial decisions, some of the most stressful, complex and impactful ones we make, provide a useful tool for this level of inquiry. In this paper, we claim that the negative consequences of stress on financial decision making can be understood, and potentially mitigated, through the lens of psychological distancing. To test this idea, we test levels of financial stress, attitudes and behaviors in a sample of 1011 Americans, showing that higher levels of stress correlate with a number of poor financial attitudes and behaviors. This effect persists even when controlling for household income and credit score. In an effort to understand what strategies can thwart these effects, we find that more abstract (high-level construal) thinking styles are associated with positive financial behaviors and practices. Finally, we show that an abstraction exercise that induces highlevel construal can have a remediating effect, improving certain financial behaviors and attitudes, even without addressing stress levels directly.
\end{abstract}

Keywords: decision making; finance; behavioral economics; behavioral finance; stress; construal level; psychological distance

The research presented here was generously supported by Capital One Financial Corporation as part of efforts to improve the financial wellbeing of Americans. In addition, we are thankful to Dr. Hans Melo, Dr. Alireza Hashemi, Dr. Tomasz Gajderowicz and Stephen Shu for their support in editing this document as well as The Decision Lab's advisor network for providing feedback on the practical translations of the research.

\section{INTRODUCTION}

\section{I.1. Context}

Stress has become an extremely pervasive and persistent issue in the US and around the world. Despite aggressive growth in the wellness industry - between 20152017 , the global wellness economy grew from $\$ 3.7$ trillion to a $\$ 4.2$ trillion market, which is nearly twice as fast as global economic growth (Global Wellness Institute 2018) - stress levels still continue to rise. For the first time in its 10-year history, the 2017 American Psychological Association survey on stress demonstrated a statistically significant increase in stress levels across the United States from the previous year (American Psychological Association, 2017). Specifically, the report indicated that $75 \%$ of American adults have experienced at least one symptom of stress in the past month (American Psychological Association, 2017), 57\% of Americans report being "paralyzed by stress" and $1 / 3$ reported having visited a doctor for symptoms relating to stress (Maureen and Margot).

a Corresponding Author: brooke@thedecisionlab.com
The single biggest source of stress, according to the APA survey, is money. Given that financial attitudes and behaviors are highly impactful on financial wellbeing, it is highly likely that this stress is mediated by the quality of financial decision making (Dilmaghani 2017).

What is less clear, however, is the reverse: what effect might stress have on financial decisions?

\section{I.2. Stress Reallocates Cognitive Resources and Induces Bias}

While past research has demonstrated correlations between stress and a number of cognitive functions, such as cognitive capacity and working memory (Lupien et al. 2007; Wolf 2009), its effects on decision making can best be understood in the context of cognitive resource allocation. The physiological stress response system (SRS), also known as the "fight-or-flight" response, evolved as a survival mechanism that signalled to humans and other mammals to quickly respond to life-threatening situations. A situation is appraised as threatening when there is an imbalance between demands (stressors) and resources. In other words, stress occurs when a situation is perceived to be more dangerous, difficult or painful than the resources that are available to overcome it (Meurs and Perrewé 2011).

In order to compensate, the body responds by aligning resources to meet those demands. From a physiological point of view, this means that, when confronted with a dangerous situation, the hypothalamic-pituitary-adrenal axis (HPA) releases hormones such as cortisol, adrenaline and noradrenaline that create a "fight" (confrontation) or "flight" (evasion) response (Ulrich-Lai and Herman 2009). This, in turn, causes a rise in heart rate, blood pressure 
and breathing rate as well as a decrease in immune activity and digestion (Stephens and Wand 2012). This nearinstantaneous physiological response helps us to fight the threat or flee to safety. Most of the stress people encounter is acute stress and is caused by isolated incidents such as a fast run down a ski slope or an exam. In these scenarios, stress can be beneficial to meeting the demands of the situations. In contrast, chronic stress is a constant low-level stress and while it is less demanding than acute stress, over time the persistent activation of the HPA can wreak havoc through long-term attrition. Because physical and mental resources are consistently being depleted, it can cause long-term health problems over time, such as increased blood pressure, risk of heart attacks, weight gain and depression.

A similar process occurs in a psychological context. When faced with stress, people adapt by falling back on strategies with lower cognitive cost, adopting a blunted sensitivity to new information about future rewards and generally engaging in unsystematic and hurried decision making (Porcelli and Delgado (2017), 2017; Janis \& Mann, 1977). This effect has been robustly demonstrated under a variety of experimental paradigms, such as reduced reinforcement contingencies during a signaldetection paradigm (Bogdan and Pizzagalli 2006; Bogdan et al. 2011; Pizzagalli et al. 2007) and a reversion towards habitual response patterns following stress and these are resistant to changes in external (e.g. environmental context) or internal conditions (e.g. satiety; Dias-Ferreira et al. (2009); Lemmens et al. (2011); Schwabe and Wolf (2009)). Research has also demonstrated how the stress response alters decision-making performance under uncertainty (Leder et al., 2013; (Starcke and Brand 2012)), such as those we make on a daily basis about our finances. Specifically, research has suggested that stress impairs executive control (Hermans et al., 2014), causes a shift from goal-directed to habit driven behaviors (Soares et al. 2012) and induces attentional narrowing by focusing on the immediate circumstances to prevent irrelevant information from interfering (Norman and Shallice, 1986).

There are a few evolutionary reasons why stress causes a shift in the application of cognitive resources (e.g. attention, executive control, and temporal orientation). By limiting the scope of application of cognitive functions, stress mobilizes mental resources to focus on solving a problem quicker and more efficiently- which is beneficial for survival. This strategy aligns well with optimizing cognitive resources to overcome stressors within a specific context, but may not necessarily be the most beneficial strategy for the long-term self. This cognitive dissonance between the present and future self is a mismatch that has spurred much of the research done in behavioral economics and behavioral finance and yielded a myriad of insights on cognitive biases that seemingly betray the tenets of rational choice (Kahneman 2003). Temporal discounting is one bias that has been studied extensively in the context of behavioral finance as it describes the tendency to discount rewards as they approach the temporal horizon in the future or in the past, whereas rewards in the present are assessed at full value. Temporal discounting and the limited application of cognitive functions under stressful situations explains why people make sub-optimal financial decisions that do not always align with their long-term financial goals.

\section{I.3. Reallocation of Cognitive Resources Changes Psychological Distance}

The reallocation of cognitive resources that occurs as a consequence of stress can also be understood as a change in psychological distance - i.e. the perceived difference between a person and elements of their environment (people, places, moments, objects, ideas, etc.) within their mental model of the world (Trope and Liberman 2010). As an illustrative example, let us consider the case of John and Jane, two acquaintances. Let us say that John feels a medium psychological distance to Jane - i.e. he does not really know her but feels that they are potentially alike and may get along if they spent more time together. Now, let us say that John's stress level increases. Under the narrowing-of-attention model of stress, this would cause cognitive resources to be allocated to his immediate context, thus increasing the psychological distance between him and things outside of that context, such as members of his outer social circle. This would in turn result in a larger psychological distance to Jane, potentially causing John to now feel that she is a stranger and someone that may be a threat.

The dimensions of psychological distance (space, time, social and hypothetical) are closely related to attention and thus provide an interesting lens in understanding how stress might affect financial decisions. For example, by asking people to think about who they would spend time with in their retirement prompts people to think about their future with a lower psychological distance and doing this was effective in getting recipients of the messaging to take steps towards retirement planning (Ontario Securities Commission 2018). In the context of financial decision making, attentional narrowing may skew decisions to the present moment (as much of the research in behavioral economics and behavioral finances shows is the case) and thus have serious negative consequences on people's ability to save for retirement, pay off their debts and achieve long-term financial health (Haushofer et al. 2015). The link between stress and psychological distancing raises the interesting possibility that poor financial practices are locked in a vicious circle: financial decisions cause stress, which in turn increases psychological distancing and makes us worse at making financial decisions. The first goal of our study is to test whether this is indeed the case. 


\section{I.4. Construal Level Theory in decision making contexts}

Psychological distance is a component of a broader direction of inquiry termed Construal Level Theory (CLT), which provides a framework to understand how level of psychological distance can influence decision making in different contexts. According to CLT, the same event or action can be represented at different levels of psychological distance (Freitas et al. 2004):

\begin{tabular}{|l|l|}
\hline High-Level Construal & Low-Level Construal \\
\hline Predominantly abstract & Predominantly concrete \\
\hline General & Specific \\
\hline Desirability - i.e. the "why" & Feasibility - i.e. the "how" \\
\hline $\begin{array}{l}\text { Distant in time, space or } \\
\text { social environment }\end{array}$ & $\begin{array}{l}\text { Near in time, space or social } \\
\text { environment }\end{array}$ \\
\hline $\begin{array}{l}\text { Purposive aspects of an } \\
\text { action }\end{array}$ & Tangible aspects of an action \\
\hline
\end{tabular}

Table I. The difference between high-level and low-level construal, as defined by Construal Level Theory

CLT is a useful framework to understand why people fail to act on their intentions/goals, despite having the knowledge, skill and opportunity required to do what they want. Research on self-control and motivation have attempted to answer these questions, but a more granular approach - that targets the way people think about their goals and decisions - has shown more promise in changing behaviour in different contexts. Several studies have shown that construal level mediates decision making by influencing self-control, future discounting, risk perception, choice confidence and motivation (Chiou et al. 2013; Fujita et al. 2019; MacGregor et al. 2017; Tsai and McGill 2010).

In particular, inducing a high-level construal mindset was associated with improved decision making in a number of ways: it correlated with lower smoking rates (Chiou et al. 2013), lower consumption of unhealthy food (Price, Higgs, \& Lee, 2016), and increased willingness to save (Fujita et al. 2006; Rudzinska-Wojciechowska 2017). In situations where self-control would be tested, focusing on an abstract version of a scenario by extracting highlevel features appears to be more useful than focusing on low-level, subordinate features. Simply, by focusing on what the goal means, rather than all the details and obstacles that may arise, is more constructive and enables people to follow through on their intentions more reliably.

According to the literature, high-level construal can shift the mode of thinking back to a more deliberative system that helps reduce harmful habit-driven behaviors (Chiou et al. 2013; Fujita et al. 2006; Price et al., 2016; Rudzinska-Wojciechowska 2017).
High level construal leads to a decreased preference for immediate outcomes (over delayed outcomes) and increased intention to exert self-control, as well as less positive evaluations of any temptations that could undermine self-control (Fujita et al. 2006). In a study where regular smokers received high-level construal manipulation, they were more likely to refrain from smoking; subsequently, they smoked fewer cigarettes (Chiou et al. 2013). By mobilizing the deliberate system, the individual is actively inhibiting the automatic system that prevents them from following through on their actions. Under stressful situation, this shifting from automatic to deliberate mode of thinking can be leveraged to counteract the reduction of psychological and biological resources that occurs when faced with a stressor.

Building on what is currently known about construal level and decision making, the second goal of this study is to test whether construal level is generally associated with difference in financial decision making. The third goal of this study will be to test whether manipulating construal level can reduce biases associated with financial attitudes and behaviors.

\section{I.5. The Present Study: Construal Level Manipulation as a Way to Disarm Stress-Induced Decision Bias}

Based on the literature reviewed above, the present study aims to test has three key hypotheses:

1. Financial stress correlates with poorer financial behaviors and attitudes

2. High-level construal is associated with positive financial behaviors and attitudes

3. Engaging in a short mental task known to raise construal level can have significant positive effects on financial behaviors and attitudes.

\section{METHODOLOGY}

A sample of 1011 participants from the United States were recruited through Amazon Mechanical Turk. After giving informed consent, participants were assigned using a pseudo-random number generator to one of the three conditions of the experiment: a high-level construal condition, a low-level construal condition, and a control condition. Following this construal level manipulation task, participants completed a series of questionnaires aimed at investigating financial attitudes and behaviors, a series of questionnaires aimed at investigating their general and finance-specific stress levels, and demographic questionnaires (more detailed methodology is provided in Appendix A, available upon request). The complete flow of 
the tasks is visualized in the figure below, with the three conditions shown after the Onboarding stage.

\section{II.1. Construal level manipulation}

Construal level was manipulated in the experimental conditions (not the control condition) using a 'Why' task and a 'How' task adapted from Freitas et al. (2004); participants assigned to the high-level construal group were asked to supply four reasons why people save money, whereas participants assigned to the low-level construal group were asked to supply four ways how people save money. As noted above, this task was administered at the outset of the questionnaire. The control group did not complete either task and proceeded immediately to the subsequent tasks.

There were also four recalls administered periodically throughout the exercise to the experimental groups (none for the control group). During the first recall, the participants were asked to elaborate further on the first point they supplied about reasons why/ways how people save money; in the second recall, they were asked to elaborate on their second point, and so on. The recalls were intended to reinforce the construal level manipulation, and follow an adaptation of the designs of Freitas et al. (2004) and (Rudzinska-Wojciechowska 2017).

\section{II.2. Financial decisions}

The financial attitudes and behaviors of the participants were measured using a series of tasks. First, they filled out the financial attitudes questionnaire, which consists of a series of 25 statements aimed at assessing attitudes towards finances and financial behaviors. Statements included "Finances control my life," "Success will come to those who work hard," and "Credit cards are a tool to build credit." Each statement was rated on a five-point Likert scale from $1=$ strongly disagree to $5=$ strongly agree.

Participants then completed a budgeting task (adapted from Rudzinska-Wojciechowska (2017)) which was aimed at investigating whether a construal-level manipulation changes the amount of money put towards savings, as was found in Rudzinska-Wojciechowska (2017). They were first asked to write down their approximate total monthly spending. They were then asked to provide an estimate of how much of that total budget would be spent on the following categories: 1) Essential expenses, 2) Non-essential expenses, 3) Savings, and 4) Paying off debts. The percentage of total money spent on each category were used as dependent variables.

Next, participants completed a windfall spending task adapted from (Rudzinska-Wojciechowska 2017), where they were asked to imagine a scenario where they received an unexpected gift of $\$ 1000$ USD. This task was intended to be a replication of (Rudzinska-Wojciechowska 2017) which showed that participants were more likely to put more money towards savings when encouraged to think about why they should save money (as opposed to how). They were asked to provide an estimate of how much of that total amount they would spend on 1) Essential expenses, 2) Non-essential expenses, and 3) Pay debts / savings. The amounts of money spent on each category were used as dependent variables.

Participants then completed a delay-discounting task aimed at investigating self-control, adapted from Fujita, Trope, Liberman, and Levin-Sagi (2006). Previous studies have found that high construal level increases selfcontrol, which leads to less delayed discounting (Fujita et al. 2006; Rudzinska-Wojciechowska 2017). Delaydiscounting is also affected by stress (Diller et al. 2011; Starcke and Brand 2016). The task asked participants to provide a dollar amount for receiving the following items, both now and at a delayed time: 1) A new phone, 2) A set of four movie passes, 3) A gift certificate to a favourite restaurant, and 4) A gift certificate to Amazon purchases. The delays for each of the respective items were 1) One year from now, 2) One month from now, 3) Six months from now, and 4) One year from now, following the order of Fujita et al. (2006). The difference between the immediate and delayed amounts for each item were used as the dependent variable to measure future discounting.

Next, participants answered six questions aimed at assessing (within-participant) framing bias, adapted from Mahoney, Buboltz, Levin, Doverspike, and Svyantek (2011)'s three gain-framed problems: G1, G3, G5, and three equivalent loss-framed problems: L1, L3, L5. For each problem, a score of 1 was given when the participant chose the more risky option and 0 for the sure-thing. Two composite outcomes created from these item scores were used as dependent variables: the sum of the gain-framed problems, the sum of the loss-framed problems.

Participants then completed a test assessing optimism bias related to financial outcomes, adapted from Weinstein (1982). They were asked the following three questions: Compared to someone in a similar situation to your own, how likely is is that you will 1) Retire by age $65 ?, 2)$ Own your own home worth over $\$ 300,000$ in your lifetime?, and 3) Earn over $\$ 100,000$ per year in your lifetime? Responses were given on a 7-point Likert scale from: 1) Much less likely than most people, 4) Average for most people like me, to 7) Much more likely than most people. These questions were intended to assess whether stress and construal level are related to optimism about common American financial aspirations.

Finally, participants completed an anchoring bias test, adapted from Tversky \& Kahneman (1974), as stress has been shown to influence anchoring and adjustment bias (Starcke \& Brand, 2016). The difference between the arbitrary anchor (the last two digits of the participant's 


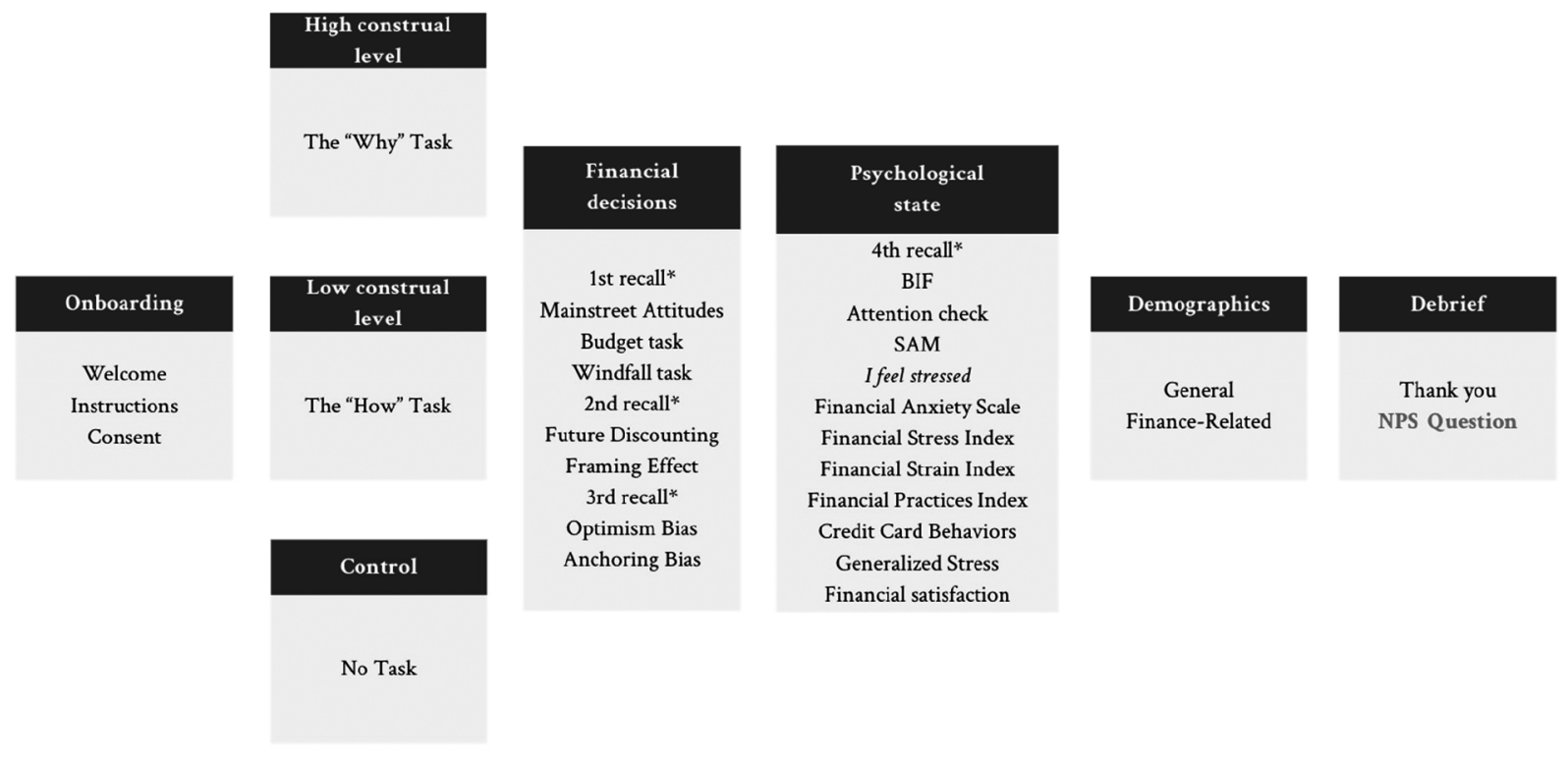

Figure 1. Overview of the experimental design

*Recall tasks were administered only to the high-level construal and low-level construal groups; no recall tasks were administered to the control group.

phone number) and their own estimate of the percentage of African countries in the UN was used as a dependent variable.

\section{II.3. Psychological states}

In the first test of this block, participants filled out the Behavior Identification Form (BIF; Vallacher, R. R. \& Wegner, D. M., 1989). The BIF is a measure of level of construal and is sensitive to construal-level manipulations (Fujita et al. 2006; Liberman \& Trope, 1998). Higher scores on the BIF ( $\max 25)$ indicate a higher level of construal (following Fujita et al. 2006). Participants then completed an attention-check in order to screen out inattentive participants.

Participants next filled in the self-assessment manikins (SAM) used to assess the emotional dimensions of valence, arousal, and dominance along a nine-point scale (Bradley and Lang 1994). These scales were used to measure the current affective state of the participants, which were not explicitly related to financial hardship or more 'objective' measures of life stress, discussed further below. This would allow us to examine the relationship between stress, construal level, and raw emotions. During each assessment, participants were presented with the graphic image of the scale along with the instructions of Imbir (2016). Following the three SAM scales, participants were asked to rate how strongly they agreed with the following statement on a 5-point likert scale: "I feel stressed right now" ( $1=$ strongly disagree, $5=$ strongly agree; i.e. the Momentary Stress Scale).
In order to assess financial anxiety and financial stress, participants then completed the Financial Anxiety Scale (FAS; Archuleta, Dale, \& Spann, 2013) and the Financial Stress Scale (FSS) adapted from Hayhoe, Leach, Turner, Bruin, and Lawrence, 2000. Rather than the original binary scores, participants were asked to report how strongly they agree to the seven FSS statements on a scale of 1 (strongly disagree) to 5 (strongly agree); the sum of the scores being indicator of financial stress. After these, the participants completed the Financial Strain question (Feather, 1989).

The Financial Practices Index (FPI) (Hayhoe et al. 2000) was the next task for the participants. The FPI consists of 10 questions investigating various aspects of financial stability. Participants were asked to rate how often they engage in behaviors such as writing budgets, saving on a regular basis, making minimum payments, etc. Each question was on a scale of 1) Never to 4) Almost always, with the sum of each answer serving as an index of healthy financial practices.

Participants were then asked three questions about credit card ownership: 1) How many credit cards do you have which carry a balance? 2) How many credit cards do you have that are charged to the credit limit? 3) How many credit cards do you have in total?. Answers were scored following Hayhoe et al. (2000).

Participants then completed the Perceived Stress Scale (PSS; Cohen, Kamarck, \& Mermelstein, 1983), with a higher PSS score (maximum 56) indicating higher general life stress. They then completed the Financial Satisfaction scale (Robb and Woodyard 2011), with higher 
scores (max 10) indicating higher satisfaction with their financial situation.

\section{II.4. Demographics}

Finally, participants completed a series of demographic questions. They reported their age, sex, marital status, household income (in $\$ 10,000$ brackets from $\$ 0$ to $>\$ 100,000)$, whether they rent or own their home, whether they rent, lease or own a car, their highest level of education achieved, and their FICO credit score (in 50 unit brackets from 300 to $>800$, including an option for 'I don't know'). Participants were also asked to rate the questionnaire itself for how likely they would be to recommend it to a friend on a scale of 1 to 5 , known as the "Net Promoter Score."

\section{RESULTS}

\section{III.1. Approach to Analysis}

Of the total sample of 1011 participants, 47 were removed for failing the attention check, failing to follow instructions, or providing nonsensical answers, resulting in a valid sample of 964 participants. Descriptive statistics of pertinent sample characteristics are reported first, then zero-order and partial correlations. Then a multivariate canonical correlation and analyses of variance examining the effect of the BIF and construal level manipulation on outcome variables are reported.

In order to satisfy some of the assumptions of the significance tests involved, univariate and multivariate outliers were removed. Univariate outliers were those with a $z$-score of $> \pm 3.0$. Multivariate outliers were those with where the Chi-square test of Mahalanobis distance was significant at $p<.001$. For this report, results are reported with outliers removed, unless mentioned otherwise.

\section{III.2. Descriptive Statistics}

As often seen in MTurk studies, the sample tended to be younger, more educated and less affluent than the average American, but was otherwise representative of a general American population (Paolacci and Chandler 2014). The sample showed a relatively even gender split with 514 males $(53.3 \%)$ and a mean age of 37.41 years $(S D=11.54, N=963)^{1}$. Nearly half $(47.20 \%)$ of the

\footnotetext{
${ }^{1}$ Outliers were not removed for descriptive statistics.
}

sample was single, $40.60 \%$ were married, and the remaining participants were either divorced, common-law or widowed. Most of the participants $(55.80 \%)$ had a Bachelor's degree or higher. The mean numeric FICO score was $669.13(S D=119.13, N=834)^{2}$. Mean numeric income was $\$ 45,638(S D=\$ 24,355, N=846)^{3}$. This is less than the American mean income of $\$ 72,641$ in 2018 (median $=\$ 61,372$; US Census Bureau, 2018).

The participants appeared to be under a fair amount of general life stress: $30 \%$ reported feeling nervous and "stressed", and $58 \%$ reported not being able to cope with all the things they had to do. In terms of financial stress specifically, $34 \%$ of those surveyed reported feeling anxious about their financial situation, $58 \%$ feeling that finances control their life and $19.6 \%$ having difficulty controlling their money-related worries. The biggest financial worries were about having enough money to retire (68\%), keeping up with the cost of living (56\%) and managing debt levels $(45 \%)^{4}$.

In sum, our sample appeared to be representative of the American population and under considerable stress, in the context of both life stress generally and financial stress specifically.

\section{III.3. Financial Anxiety}

As a next step in our data analysis, we ran a correlation analysis. Unsurprisingly, scores in the financial anxiety scale (FAS) tend to correlate inversely with income and FICO scores. Given that we were not interested in the effects of financial situation (but rather just the stress), we controlled for numeric income and numeric FICO scores through partial correlation, which allowed us to isolate the relationships between FAS and the other psychological variables in our study.

Higher FAS was associated with more agreement with a number of attitudes such as: 'Finances control my life,' 'I worry about being able to keep up with the cost of living,' and 'When I get my paycheck, I want to spend it even though I know I shouldn't,' with more disagreement with the statement 'Success will come to those who work hard.' The findings went beyond the financial attitudes questions as well. Participants higher on the FAS also reported lower levels of financial satisfaction, scored lower on the financial practices index, and scored higher on the financial strain, financial stress and PSS scales. They also had more credit cards that carry a balance, more credit cards charged to the limit, and more credit cards in total.

\footnotetext{
${ }^{2}$ Excluding participants who reported not knowing their FICO score.

${ }^{3}$ Excluding participants who earned $>\$ 100,000$.

${ }^{4}$ Financial attitudes, FAS, and PSS question scores $>$ midpoint.
} 
Even controlling for income and credit score, with increases in financial stress, poor financial attitudes and behaviors become more prevalent while healthy financial attitudes and behaviors become less prevalent. Looking beyond financial outcomes to psychological states overall, participants with higher FAS also reported lower levels of pleasant emotions (emotional valence) lower levels of feeling in control (dominance), and higher levels of momentary stress ('I feel stressed right now'). Partial correlations between FAS, BIF (our main measure of construal level) and key variables in the study are reported in Table 2. Interestingly, the negative financial attitudes and behaviours that correlate with FAS tend to correlate in the opposite direction with BIF, indicating that level of construal may mediate the negative effects of financial stress.

This analysis answers the first question of the study: financial stress takes a huge toll on our financial attitudes and behaviors, and on our psychology more generally. These effects of financial stress are also independent from income or credit score, which themselves have important effects (e.g.: Santiago, Wadsworth, \& Stump, 2011) but are not at issue here.

\section{III.4. Observed Construal Level}

Recall that the second goal of the study was to determine whether high-level construal is a significant predictor of positive financial behaviors and attitudes. A multivariate canonical correlation was conducted to examine the relationship between BIF (our main measure of construal level) and the outcome variables of the study; this statistical approach bundles all of the outcome variables together into a single package, ${ }^{5}$ using dimensionality reduction to control for correlation between all of these individual variables. Running a single test on this package as a whole, rather than multiple tests for the variables individually, addresses concerns about Type 1 error and the correlations between dependent variables (Sherry \& Henson, 2005).

The analysis showed that a higher BIF score (i.e. highlevel construal) was a significant omnibus predictor of the outcome variables (Wilks $\lambda=.80, F(65,416)=1.58$, $p=.005$, Eigenvalue $=.25$, canonical correlation $=.445$, squared canonical correlation $=.20) ;{ }^{6}$ that is to say, BIF score is a predictor of the outcome variables when

\footnotetext{
${ }^{5}$ When building the Financial Practices questions from Hayhoe, Leach, Turner, Bruin, and Lawrence (2000) into the package, they were included individually rather than summed as the original authors did.

6 The BIF model with the aggregate financial practices index was also significant, Wilks $\lambda=.84, F(56,523)=1.76, p=.001$, Eigenvalue $=.19$, squared canonical correlation $=.16$.
}

bundled together into the single package. Looking at which individual elements within the multivariate package of outcomes were the main drivers of the effect, there is a positive association between BIF several measures of positive financial attitudes and behaviors (Table III). When examining which dependent variables correlated most strongly with the single multivariate dependent variable package (those with structure coefficients $> \pm .3$; the strongest drivers of the effect), it appears that highlevel construal is most highly related to financial satisfaction, positive emotional valence, saving money and feeling in control of finances, whereas low-level construal is most highly related to financial stress, general life stress, and spending a paycheck even though they know they shouldn't. A full list of the standardized canonical function coefficients (function weights) and structure coefficients (the correlation between the individual dependent variable and the multivariate package) are reported in Appendix B (available upon request). The significant univariate dependent variables predicted by $\mathrm{BIF}^{7}$ (along with their partial correlations, controlling for numeric income and FICO scores) are reported in Table II.

In brief, these analyses suggest that abstract thinking (high BIF scores, high construal level) has significant positive effects on a broad range of financial attitudes and behaviors, whereas more concrete thinking (low BIF scores, low construal level) is connected with negative outcomes. Thus, it seems that those with higher-level construal have more positive emotions, satisfaction with their finances and life situation, and more healthy financial attitudes and practices. This modeling approach allows us to measure the effect of construal level while controlling for income level and FICO score by including it in the multivariate dependent variable package, two factors that are known to have a strong influence on financial attitudes and behaviors.

One interesting note here is that construal level is negatively associated with perceived stress. That is to say, thinking abstractly tends to be connected with lower stress, whereas thinking concretely tends to be connected with higher stress. This finding highlights the potential relevance of psychological distance to the issues discussed here. If psychological distance is a key determining factor in effective decisions about longer-term topics (such as financial decisions), that would explain both why stress decreases performance (by decreasing psychological distance, tightening the mind's focus on the immediate) and why high construal level increases performance (by increasing psychological distance, loosening the mind's focus from the immediate). Their respective relationships to psychological distance suggest a nuanced, textured relationship between stress and construal level.

\footnotetext{
7 That is, the univariate $F$ tests with BIF as the predictor, where $p<.05$
} 


\begin{tabular}{|c|c|}
\hline \multicolumn{2}{|l|}{ Factor } \\
\hline \multicolumn{2}{|l|}{ Positive emotional valence } \\
\hline \multicolumn{2}{|l|}{ Dominance } \\
\hline \multicolumn{2}{|l|}{ Momentary stress } \\
\hline \multicolumn{2}{|l|}{ Perceived stress scale } \\
\hline \multicolumn{2}{|l|}{ Financial satisfaction } \\
\hline \multicolumn{2}{|c|}{ I can enjoy my life because of the way I manage my money } \\
\hline \multicolumn{2}{|c|}{ I can handle a major unexpected expense } \\
\hline \multicolumn{2}{|c|}{ I feel in control of my finances } \\
\hline \multicolumn{2}{|l|}{ I worry about getting out of debt } \\
\hline \multicolumn{2}{|c|}{ I worry about being able to keep up with the cost of living } \\
\hline \multicolumn{2}{|c|}{ Success will come to those who work hard } \\
\hline \multicolumn{2}{|c|}{ I find it important to monitor my spending for small unexp } \\
\hline \multicolumn{2}{|c|}{$\begin{array}{l}\text { Credit cards are meant to be used to cover unexpected exp } \\
\text { income }\end{array}$} \\
\hline \multicolumn{2}{|l|}{ Write a budget } \\
\hline \multicolumn{2}{|l|}{ Save on a regular basis } \\
\hline \multicolumn{2}{|c|}{ When I get my paycheck, I want to spend it even though I } \\
\hline \multicolumn{2}{|c|}{$\begin{array}{l}\text { Table II. Key univariate dependent variables predicted by Be } \\
\text { Financial Anxiety Scale, Behaviour Identification Form, aft }\end{array}$} \\
\hline Dependent Variable & $\begin{array}{l}\text { Structure Coefficient } \\
\text { (Correlation) }\end{array}$ \\
\hline Financial satisfaction & 0.375 \\
\hline Emotional valence & 0.373 \\
\hline Budgeting task - savings & 0.342 \\
\hline I feel in control of my finances & 0.301 \\
\hline Financial stress scale & -0.305 \\
\hline Perceived stress scale & -0.343 \\
\hline $\begin{array}{l}\text { When I get my paycheck, I } \\
\text { want to spend it even though } \\
\text { I know I shouldn't }\end{array}$ & -0.424 \\
\hline
\end{tabular}

Table III. Strongest correlations (structure coefficients) between the dependent multivariate package and individual dependent variables; the biggest drivers of the multivariate effect.

\section{III.5. Manipulated Construal Level}

Finally, turning to the third goal of the study, this portion of the analysis aims to assess the effectiveness of a candidate construal-level manipulation on financial attitudes and behaviors. The preceding section concluded that construal level does have an effect; what is being examined here is whether that construal level can be changed with an intervention that will prompt an improvement in financial decision making. Two analyses were conducted here, both applying Multivariate Analy-

Financial Anxiety Construal Level

\begin{tabular}{|c|c|}
\hline-0.337 & 0.177 \\
\hline-0.191 & 0.113 \\
\hline 0.487 & -0.121 \\
\hline 0.638 & -0.118 \\
\hline-0.526 & 0.151 \\
\hline-0.445 & 0.105 \\
\hline-0.48 & 0.115 \\
\hline-0.467 & 0.11 \\
\hline 0.534 & -0.114 \\
\hline 0.486 & -0.074 \\
\hline-0.269 & 0.106 \\
\hline 0.009 & 0.103 \\
\hline 0.027 & 0.119 \\
\hline-0.054 & 0.116 \\
\hline-0.387 & 0.109 \\
\hline 0.272 & -0.183 \\
\hline
\end{tabular}


model with experimental condition as the predictor (i.e., the 'Why' task condition, the 'How' task condition and the control condition) showed statistically significant results (Wilkes $\lambda=.79, F(132,1372)=1.32, p=.011$, partial $\left.\eta^{2}=.113\right)$. Given that there were three experimental groups, the dimension reduction analysis produced two discriminant functions, orthogonal to each other. The first discriminant function explained slightly more than half of the variance between the two functions, Eigenvalue $=0.134$, percent of variance $=52.74$, canonical correlation $=0.344$, squared canonical correlation $=.118$ with the second discriminant function explaining slightly less than half, Eigenvalue $=0.120$, percent of variance $=47.26$, canonical correlation $=0.328$, squared canonical correlation $=.108$. Only the omnibus analysis which considered both discriminant functions simultaneously was significant (reported above), with the second function alone reaching marginal significance, Wilkes $\lambda=.89, F(65,687)=1.27, p=.08$. The standardized discriminant function coefficients (weights) along with the correlations between the dependent variables and the discriminant functions (coefficients) are reported in Appendix D (available upon request). None of the dependent variables correlated with the first discriminant function above $r \pm .3$. For the second discriminant function, the strongest correlations were with the variables 'I can handle a major unexpected expense' $(r=.371)$ and 'I feel in control of my finances' $(r=.315)$.

A follow-up Roy-Bargman Stepdown $F$ test was performed to test hypotheses about the effects of the manipulation on our dependent variables (Roy, 1958). This test is similar in concept to a hierarchical regression, where the variance associated with each variable is systematically accounted for on a step-by-step basis (Finch, 2007). The primary interest was whether the three groups differed on their BIF scores, after controlling for differences in income and FICO scores. Secondarily, the financial anxiety and perceived stress scales were taken to be key variables associated with construal level that would be important to show changes due to a construal-level manipulation. Finally, we tested the other dependent variables shown to be predicted by BIF in the above analysis, in the following order: financial stress scale, financial satisfaction, emotional valence, budgeting on savings, agreement with 'I feel in control of my finances', and agreement with 'When I get my paycheck, I want to spend it, even though I know I shouldn't'. The results of the analysis which included outliers are reported in Appendix C (available upon request), and show that the groups differed on BIF $(p<.05)$, with those in the 'Why' task showing the highest average BIF scores. Emotional valence was marginally significant $(p=.097)$, with those in the 'How' task showing the highest emotional valence. Finally, agreement with the statement 'I feel in control of my finances' was significant $(p<.05)$, with those in the
'Why' task condition showing the most agreement with the statement.

When the effects of the experimental condition are examined on individual dependent variables in isolation ${ }^{11}$, our results indicate that the high-level construal manipulation yielded:

\begin{tabular}{|l|c|}
\hline Factor & Significance \\
\hline Higher reported savings practices & $p<.05$ \\
\hline Higher reported budgeting practices & $p<.05$ \\
\hline $\begin{array}{l}\text { Higher reported ability to handle unex- } \\
\text { pected expenses }\end{array}$ & $p<.05$ \\
\hline
\end{tabular}

Table IV. List of effects yielded by the high-level construal manipulation.

Interestingly, those in the 'How' condition also showed improvements on some dimensions - namely on the likelihood of spending windfall profits on essentials versus non-essentials $(p<.05)$. This finding suggests further nuance to our understanding of how construal level affects financial decision making. While the overriding picture suggests that abstract thinking may be beneficial for financial decision making in general, there may also be specific components of financial behavior that benefit from concrete thinking. This would suggest that financial health is benefited by the right mix of abstract and concrete thinking; the appropriate style of thinking should be chosen based on the task at hand, acknowledging that financial tasks are more often benefited by abstract thinking but that concrete thinking also has its role to play. Accordingly, an intervention to support consumers in adopting the right mindset might need to be slightly more dynamics, supporting consumers in adopting the right mindset for the task at hand.

In sum, what do these analyses show regarding the the efficacy of a construal-level manipulation to affect financial decision making (which was the third goal of our study)? While some effects were visible under certain modeling conditions, those effects were not broad-based and robust across methodological approaches, suggesting that the intervention was not strong enough to be considered mature.

The findings here show a connection between the intervention and construal level, and the findings from the canonical correlation analysis above show a connection between construal level and key outcomes. However, if we measure from the intervention to the key outcomes, we can measure traces but not a clear and reliable signal. A stronger intervention should yield a signal that can be read clearly even through the noise. While more ad-

11 These results refer to univariate $F$ tests when outliers were included in the model with disaggregated financial practices. 
vanced statistical analyses might have discerned the signal even here, these more powerful tools represent better filters for noise, but ultimately such strong filters should not be necessary if the signal is clear - which is the mark of a mature intervention, not an early-stage test such as this one.

\section{DISCUSSION}

This study had several goals. The first was to measure the effects of financial stress on financial attitudes and behaviors, and the study demonstrated that these effects are considerable and negative, even when controlling for income and credit score. Most notably, we found that higher levels of stress correlate with poorer financial practices, lower reported saving and budgeting rates, poorer mood, lower feelings of control over life and finances, and lower levels of agreement with the idea that success comes to those who work hard.

The second goal of the study was to assess the effects of construal level (i.e. abstract vs. concrete thinking) on those same financial attitudes and behaviors, and the study demonstrated that more abstract thinking has more or less an opposite effect on financial attitudes and behaviors compared to stress. Most notably, we found that high-level construal predicted higher reported savings and budgeting rates, lower stress, higher feelings of control over finances and higher satisfaction with finances. This finding raises the interesting possibility that construal level is at least one significant mediating factor through which stress affects the quality of financial decision making. If this is true, then interventions focused on raising the level of construal could offer a viable approach for mitigating the effects of stress on financial outcomes.

With these theoretical bases established, the third goal was to assess the effectiveness of one candidate construal intervention in affecting financial attitudes and behaviors. The intervention in our study showed significant effects on several dimensions of financial attitudes and behaviors. Most notably, we found that our high-construal manipulation caused higher reported levels of saving and budgeting behaviors as well better reported ability to handle unexpected expenses. It is important to note that although we significantly managed to shift these important attitudes, a myriad of factors that were predicted by high-level construal, as measured by the BIF, were not affected by this particular intervention, possibly suggesting that a stronger intervention is needed to achieve a more significant effect. The results were not clear or definitive enough to claim that the intervention is mature and ready for deployment; however, it would have been far too optimistic to expect such outcomes at this early stage in development.

This study has established a theoretical basis for affecting positive change to financial attitudes and behaviors through construal level manipulations. It is our hope that the work here lays the foundation for subsequent work to devise and test various permutations of construal level interventions. Ultimately, the goal of this refinement would be to develop a tool that allows us to use construal level to substantially mitigate the negative effects of stress on financial decision making. Thinking abstractly can improve financial decision making. What remains is to build an effective tool to help consumers engage in that kind of thinking, something to support them in mitigating the challenges that stress creates for financial management.

The potential impacts of using construal level to empower better financial decisions, especially under stressful conditions but also within unstressed populations, is deep and broad - that is, its impact can have many downstream effects on overall financial health and can be used across the spectrum of financial wellness. Since money is one of the largest stressors for Americans, unlocking strategies that people can employ themselves can be an extremely empowering tool because it is putting the onus of control on them- and perception of control is a welldocumented mediator of response to stress (Litt 1988). Therefore, imparting people with tools will equip them with a sense of control that they can overcome financial difficulties by changing their construal level and motivating them to take control of their financial wellness.

While not fully conclusive, the findings of the study are promising and open up a wide range of future research opportunities that will clarify and deepen our understanding of how construal levels, both high and low, affect financial decision making broadly but also different kinds of financial decisions specifically (e.g. planning for retirement versus making a decision about whether to eat out for dinner). Specifically, by testing how construal level tasks can be used to improve decision making under different financial decision making scenarios, we can build tools that are both targeted towards specific contexts and widely applicable across contexts. Our long term goal is to be able to empower people to take control of their financial wellness and equip financial institutions with the information to design products and services that are aligned with research on how people make decisions and how these decisions are affected by stress.

\section{IV.1. Limitations}

While level of construal is an important variable related to financial stress and attitudes, and manipulating it is possible, the effects of its manipulation on other variables are somewhat diffuse. One explanation of these results is that the number of variables considered in this study is much larger than those of previous studies, which used a single dependent variable to measure the effect of construal level manipulation. Our study therefore necessitates a multivariate analysis to control for Type I 
error, but results in null findings if the effects of the manipulation are diffuse. Future studies can build off of the current findings by targeting only the psychological variables most susceptible to construal level manipulation.

Another caveat is that the current study did not track objective financial behavior and relied on self-reported budgets, financial practices and attitudes. While this may impact the ecological validity of our study, ethical and practical considerations prevent us from accessing objective financial behavior.

We measured the effect of BIF on a number of outcomes - including stress. High construal level is connected to lower stress, and high construal level improves financial attitudes and behaviors. Does high construal level improve financial attitudes and behaviors because it improves stress? This study focused on the potential of construal level to influence financial decision making, as a potential avenue to mitigate the effects of stress, but there was no hypothesis about whether construal level bypasses stress to affect decision making directly or via the effects of construal on stress itself. In terms of devising an effective intervention to support consumers in making better financial decisions, at first glance this question is not pressing. However, as research develops it may be necessary to clarify the mechanisms further, and the present study offers little insight on that point.

As a priority, future research can build on the present study by targeting psychological variables most amenable to influencing construal level. Although we found that construal level was a strong predictor of a wide variety of positive financial attitudes and behaviors, the development of stronger construal manipulations may yield more effective solutions to disarming the negative effects of stress on financial decision making and attitudes. Also, future research should be mindful that while this study focused on the positive effects of high construal level on the negative effects of stress, low-level construal can be constructive when developing more concrete to-do plans. Thus, in order to fully leverage findings from this literature, future studies should examine the best combination of high- and low-level construal on different aspects of financial attitudes and behavior. A future study could also use a longitudinal design which examines the impact of the manipulation across time to determine the longevity of the effect.

American Psychological Association and others. Stress in america: Coping with change. Stress in America ${ }^{T M}$ Survey. American Psychological Association, 2017.

Kristy L Archuleta, Anita Dale, and Scott M Spann. College students and financial distress: Exploring debt, financial satisfaction, and financial anxiety. Journal of Financial Counseling and Planning, 24(2):50-62, 2013.
Ryan Bogdan and Diego A Pizzagalli. Acute stress reduces reward responsiveness: implications for depression. Biological psychiatry, 60(10):1147-1154, 2006. doi: 10.1016/j.biopsych.2006.03.037.

Ryan Bogdan, Diane L Santesso, Jesen Fagerness, Roy H Perlis, and Diego A Pizzagalli. Corticotropin-releasing hormone receptor type 1 (crhr1) genetic variation and stress interact to influence reward learning. Journal of Neuroscience, 31(37):13246-13254, 2011. doi: 10.1523/jneurosci.2661-11.2011

Margaret M Bradley and Peter J Lang. Measuring emotion: the self-assessment manikin and the semantic differential. Journal of behavior therapy and experimental psychiatry, 25(1):49-59, 1994. URL https : // doi.org/10.1016/00057916(94) $90063-9$.

Wen-Bin Chiou, Wen-Hsiung Wu, and Ming-Hsu Chang. Think abstractly, smoke less: A brief construal-level intervention can promote self-control, leading to reduced cigarette consumption among current smokers. Addiction, 108(5):985992, 2013. doi:10.1111/add.12100.

Sheldon Cohen, Tom Kamarck, and Robin Mermelstein. A global measure of perceived stress. Journal of health and social behavior, 24(4):385-396, 1983. URL https ://doi.org/ $10.2307 / 2136404$.

Eduardo Dias-Ferreira, João C Sousa, Irene Melo, Pedro Morgado, Ana R Mesquita, João J Cerqueira, Rui M Costa, and Nuno Sousa. Chronic stress causes frontostriatal reorganization and affects decision-making. Science, 325(5940):621-625, 2009. doi:10.1126/science.1171203.

James W Diller, Connor HG Patros, and Paula R Prentice. Temporal discounting and heart rate reactivity to stress. Behavioural processes, 87(3):306-309, 2011. URL https : //doi.org/10.1016/j.beproc.2011.05.001.

Maryam Dilmaghani. Financial unhealthiness predicts worse health outcomes: evidence from a sample of working canadians. Public health, 144:32-41, 2017. doi: 10.1016/j.puhe.2016.11.016

Antonio L Freitas, Peter Gollwitzer, and Yaacov Trope. The influence of abstract and concrete mindsets on anticipating and guiding others' self-regulatory efforts. Journal of experimental social psychology, 40(6):739-752, 2004.

Kentaro Fujita, Yaacov Trope, Nira Liberman, and Maya Levin-Sagi. Construal levels and self-control. Journal of personality and social psychology, 90(3):351, 2006. URL https : //doi.org/10.1037/0022-3514.90.3.35.

Kentaro Fujita, Abigail A Scholer, David B Miele, and Tina Nguyen. On metamotivation: Consumers' knowledge about the role of construal level in enhancing task performance. Journal of the Association for Consumer Research, 4(1):5764, 2019. doi:10.1086/700839

Gerd Gigerenzer and Peter M Todd. Simple heuristics that make us smart. Evolution and Cognition (Paper, 1999.

Global Wellness Institute. Global wellness economy monitor. New York, 2018. URL Retrievedfromhttps : // globalwellnessinstitute.org/industry - research / 2018 global-wellness-economy-monitor/. 
Johannes Haushofer, Chaning Jang, and John Lynham. Stress and temporal discounting: do domains matter? mimeo, 2015.

Celia Ray Hayhoe, Lauren J Leach, Pamela R Turner, Marilyn J Bruin, and Frances C Lawrence. Differences in spending habits and credit use of college students. Journal of Consumer Affairs, 34(1):113-133, 2000. URL https://doi.org/ $10.1111 / j .1745-6606.2000 . t b 00087 . x$.

Kamil K Imbir. Affective norms for 718 polish short texts (anpst): Dataset with affective ratings for valence, arousal, dominance, origin, subjective significance and source dimensions. Frontiers in psychology, 7:1081, 2016. URL https: //doi.org/10.3389/fpsyg.2016.01030.

Robert-Paul Juster, Bruce S McEwen, and Sonia J Lupien. Allostatic load biomarkers of chronic stress and impact on health and cognition. Neuroscience $\&$ Biobehavioral Reviews, $35(1): 2-16,2010$.

Daniel Kahneman. A psychological perspective on economics. American economic review, 93(2):162-168, 2003. doi: $10.1257 / 000282803321946985$.

Sofie G Lemmens, Femke Rutters, Jurriaan M Born, and Margriet S Westerterp-Plantenga. Stress augments food 'wanting'and energy intake in visceral overweight subjects in the absence of hunger. Physiology \& behavior, 103(2):157-163, 2011. doi:10.1016/j.physbeh.2011.01.009.

Mark D Litt. Cognitive mediators of stressful experience: Self-efficacy and perceived control. Cognitive therapy and research, 12(3):241-260, 1988. doi:10.1007/bf01176188.

Yuanyuan Liu, Xi Zhao, and Yeyi Liu. Stress and unethical consumer attitudes: The mediating role of construal level and materialism. Personality and Individual Differences, 135:8591, 2018. doi:10.1016/j.paid.2018.06.044.

Sonia J Lupien, Francoise Maheu, Mai Tu, Alexandra Fiocco, and Tania E Schramek. The effects of stress and stress hormones on human cognition: Implications for the field of brain and cognition. Brain and cognition, 65(3):209-237, 2007.

Karen E MacGregor, Jessica J Carnevale, Nicole E Dusthimer, and Kentaro Fujita. Knowledge of the self-control benefits of high-level versus low-level construal. Journal of personality and social psychology, 112(4):607-620, 2017. doi: $10.1037 / \mathrm{pspp} 0000130$.

Kevin T Mahoney, Walter Buboltz, Irwin P Levin, Dennis Doverspike, and Daniel J Svyantek. Individual differences in a within-subjects risky-choice framing study. Personality and Individual Differences, 51(3):248-257, 2011. URL https: //doi.org/10.1016/j.paid.2010.03.035.

Connolly Maureen and Slade Margot. The united states of stress 2018 survey. Retrieved from. URL https: //www.everydayhealth.com/wellness / united-states - of stress.

Bruce S McEwen. Central effects of stress hormones in health and disease: Understanding the protective and damaging effects of stress and stress mediators. European journal of pharmacology, 583(2-3):174-185, 2008.

James A Meurs and Pamela L Perrewé. Cognitive activation theory of stress: An integrative theoretical approach to work stress. Journal of Management, 37(4):1043-1068, 2011. doi: $10.1177 / 0149206310387303$

Ontario Securities Commission. Encouraging retirement planning through behavioral insights. Retrieved from Ontario Securities Commission website:, 2018. URL https:// www.getsmarteraboutmoney.ca / resources / publications / research / encouraging - retirementplanning - through behavioral-insights/.

Gabriele Paolacci and Jesse Chandler. Inside the turk: Understanding mechanical turk as a participant pool. Current Directions in Psychological Science, 23(3):184-188, 2014. URL https : //doi.org/10.1177/0963721414531598.

Diego A Pizzagalli, Ryan Bogdan, Kyle G Ratner, and Allison L Jahn. Increased perceived stress is associated with blunted hedonic capacity: potential implications for depression research. Behaviour research and therapy, 45(11):27422753, 2007. doi:10.1016/j.brat.2007.07.013

Anthony J Porcelli and Mauricio R Delgado. Stress and decision making: effects on valuation, learning, and risk-taking. Current opinion in behavioral sciences, 14:33-39, 2017. doi: 10.1016/j.cobeha.2016.11.015

Cliff A Robb and Ann Woodyard. Financial knowledge and best practice behavior. Journal of Financial Counseling and Planning, 22(1), 2011.

Joanna Rudzinska-Wojciechowska. If you want to save, focus on the forest rather than on trees. the effects of shifts in levels of construal on saving decisions. PloS one, 12(5):e0178283, 2017. URL https : / / doi.org/10.1371/ journal.pone.0178283.

Neil Schneiderman, Gail Ironson, and Scott D Siegel. Stress and health: psychological, behavioral, and biological determinants. Annu. Rev. Clin. Psychol., 1:607-628, 2005.

Lars Schwabe and Oliver T Wolf. Stress prompts habit behavior in humans. Journal of Neuroscience, 29(22):7191-7198, 2009. doi:10.1523/jneurosci.0979-09.2009

José M Soares, Adriana Sampaio, Luís Miguel Ferreira, Nadine C Santos, Fernanda Marques, Joana Almeida Palha, João J Cerqueira, and Nuno Sousa. Stress-induced changes in human decision-making are reversible. Translational psychiatry, 2(7):e131, 2012.

Katrin Starcke and Matthias Brand. Decision making under stress: a selective review. Neuroscience $\&$ Biobehavioral Reviews, 36(4):1228-1248, 2012.

Katrin Starcke and Matthias Brand. Effects of stress on decisions under uncertainty: A meta-analysis. Psychological bulletin, 142(9):909-933, 2016. URL https ://doi.org/10.1037/ bul0000060.

Mary Ann C Stephens and Gary Wand. Stress and the hpa axi: Role of glucocorticoids in alcohol dependence. Alcohol research: current reviews, 2012.

Peggy A Thoits. Stress and health: Major findings and policy implications. Journal of health and social behavior, 51 (1_suppl):S41-S53, 2010.

Yaacov Trope and Nira Liberman. Construal-level theory of psychological distance: Correction to trope and liberman 
(2010). Psychological review, 117(3):1024-1024, 2010. doi: $10.1037 / \mathrm{a} 0020319$

Claire I Tsai and Ann L McGill. No pain, no gain? how fluency and construal level affect consumer confidence. Journal of Consumer Research, 37(5):807-821, 2010. doi: $10.1086 / 655855$

Amos Tversky and Daniel Kahneman. Judgment under uncertainty: Heuristics and biases. science, 185 (4157):1124-1131, 1974. URL https ://doi.org/10.1126/ science.185.4157.1124.

Yvonne M Ulrich-Lai and James P Herman. Neural regulation of endocrine and autonomic stress responses. Nature reviews neuroscience, 10(6):397, 2009.
US Census Bureau. Race and hispanic origin of householderhouseholds by median and mean income. Retrieved March 25, 2019, from Wikipedia website:, 2018. URL https: / / www2.census.gov / programssurveys / cps / tables / time series/historical-income-households/h05.xls.

Neil D Weinstein. Unrealistic optimism about susceptibility to health problems. Journal of behavioral medicine, 5(4):441460, 1982. URL https : //doi.org/10.1007/BF00845372.

Oliver T Wolf. Stress and memory in humans: twelve years of progress? Brain research, 1293:142-154, 2009. 